\title{
Adaptive trajectory analysis of replicator dynamics for data clustering
}

\author{
Morteza Haghir Chehreghani ${ }^{1}$
}

Received: 31 October 2015 / Accepted: 24 June 2016 / Published online: 1 August 2016

(C) The Author(s) 2016

\begin{abstract}
We study the use of replicator dynamics for data clustering and structure identification. We investigate that replicator dynamics, while running, reveals informative transitions that correspond to the significant cuts over data. Occurrence of such transitions is significantly faster than the convergence of replicator dynamics. We exploit this observation to design an efficient clustering algorithm in two steps: (1) Cut Identification, and (2) Cluster Pruning. We propose an appropriate regularization to accelerate the appearance of transitions which leads to an adaptive replicator dynamics. A main computational advantage of this regularization is that the optimal solution of the corresponding objective function can be still computed via performing a replicator dynamics. Our experiments on synthetic and real-world datasets show the effectiveness of our algorithm compared to the alternatives.
\end{abstract}

Keywords Clustering · Replicator dynamics · Cut · Transition · Regularization

\section{Introduction}

Efficient clustering plays a key role in data processing, knowledge representation and exploratory data analysis tasks such as web data analysis, image segmentation, data compression, computational biology, network analysis, computer vision, traffic management and document summarization. Often, different clustering methods minimize a cost function which penalizes inappropriate partitionings. $K$-means (MacQueen 1967) is a very common method for clustering, where its use is limited to a vector space. Other examples, which mostly perform on graph data, are Correlation Clustering (Bansal et al. 2004), Normalized Cut (Shi and Malik 2000), Pairwise Clustering (Hofmann and Buhmann 1997) and Ratio Cut (Chan et al. 1994). However, minimizing such cost functions is usually NP-hard, i.e. it requires an expo-

Editors: Thomas Gärtner, Mirco Nanni, Andrea Passerini and Celine Robardet.

$凶$ Morteza Haghir Chehreghani morteza.chehreghani@xrce.xerox.com

1 Xerox Research Centre Europe - XRCE, 6 chemin de Maupertuis, Meylan, France 
nential computational time unless $\mathrm{P}=\mathrm{NP}$. Thereby, several methods have been proposed to overcome the computational bottlenecks.

An important category of methods work based on eigenvector analysis of the Laplacian matrix. Spectral Clustering (SC) (Shi and Malik 2000; Ng et al. 2001) is the first method which exploits the information from eigenvectors. It forms a low-dimensional embedding by the bottom eigenvectors of the Laplacian of the similarity matrix and then applies $K$-means to produce the final clusters. A more recent method, called Power Iteration Clustering (PIC) (Lin and Cohen 2010), instead of embedding the data into a $K$-dimensional space, approximates an eigenvalue-weighted linear combination of all the eigenvectors of the normalized similarity matrix via early stopping of power iteration method. P-Spectral Clustering (PSC) (Bühler and Hein 2009; Hein and Bühler 2010) is another significant development which proposes a non-linear generalization of the Laplacian and then performs an iterative splitting approach based on its second eigenvector.

Another clustering approach has been developed based on performing the replicator dynamics in the context of discrete time dynamical systems and evolutionary game theory (Pavan and Pelillo 2007; Ng et al. 2012; Liu et al. 2013). Dominant Set Clustering (DSC) (Pavan and Pelillo 2007) is an iterative method which at each iteration, peels off a cluster by performing a replicator dynamics until its convergence. The method in Liu et al. (2013) proposes an iterative clustering algorithm in two steps: Shrink and Expansion. The steps help to reduce the runtime of performing replicator dynamics on the whole data, which might be slow. Then, based on the idea of Dominant Set Clustering several improvements have been proposed: Bulò et al. (2009) proposes an enumeration technique via unstabilizing specific equilibrium by increasing and making the similarity matrix asymmetric. The method in Bulò et al. (2011), called InImDyn, replaces replicator dynamics by a population dynamics motivated from the analogy with infection and immunization processes within a population of players. Another method in Hou and Pelillo (2013) proposes feature combination for classification using dominant sets.

In this paper, we investigate in detail the use of replicator dynamics for extracting clusters from the data. We observe that replicator dynamics reveals the clustering structure while running and before converging to its optimal solution. Although replicator dynamics and power iteration both compute an one-dimensional embedding of data, replicator dynamics can separate the significant structure from the noise and outliers. Thereby:

1. We analyze in detail the trajectory of replicator dynamics at different steps and show that it reveals informative transitions which provide valuable information about the cuts over the data.

2. We introduce a regularized variant of replicator dynamics which accelerates the occurrence of transitions.

3. We propose a novel clustering method that works based on detecting transitions, identifying the main cuts and then pruning the clusters.

4. We perform extensive experiments to show the efficiency of our algorithm on different synthetic and real-world datasets.

The rest of the paper is organized as following: We describe the notations and the definitions in Sect. 2. Then, we introduce the main algorithm and the adaptive replicator dynamics in Sect. 3. We investigate our method and describe the experiments in Sect. 4, and finally, we conclude the paper in Sect. 5. 


\section{Notations and definitions}

Data consists of a set of objects and the corresponding measurements which represent relations between the objects. Given a set of $N$ objects $\mathbf{O}$, the measurements can be given in different ways, e.g. as vectors or pairwise similarities. In this paper, we consider the case where the relations between the objects are given by the matrix of pairwise similarities $\mathbf{X}$.

Thereby, we represent the input by a graph $\mathcal{G}(\mathbf{O}, \mathbf{X})$ with the set of $N$ nodes (objects) $\mathbf{O}$ and the $N \times N$ symmetric and non-negative similarity matrix $\mathbf{X}$. $E$ denotes the edges of the graph, thereby $|E|$ shows the number of given pairwise similarities. Notice that vector representation can be considered as a special case of graph representation where the pairwise measurements are related to for example squared Euclidean distances (Roth et al. 2003). The goal is to partition the graph into clusters, i.e. to groups of similar objects which are distinguishable from the objects of other groups. A clustering solution is shown by $c$, where $c_{i}$ indicates the cluster label for object $i$.

We convert the pairwise similarities and distances to each other via negation and shift transformations, e.g. given pairwise distances $\mathbf{D}$, the similarity matrix $\mathbf{X}$ is obtained by

$$
\mathbf{X}=\max (\mathbf{D})-\mathbf{D}+\min (\mathbf{D}),
$$

and vice versa. This particular transformation has several properties: (1) it is nonparametric, (2) it provides an identical range for both $\mathbf{X}$ and $\mathbf{D}$, and (3) it is reversible, i.e. converting $\mathbf{X}$ (or $\mathbf{D}$ ) to $\mathbf{D}$ (or $\mathbf{X}$ ) and then again to $\mathbf{X}$ (or $\mathbf{D}$ ) gives the original matrix. In this paper, the main relevant property of this transformation is being nonparametric. Notice that if we use a kernel, e.g. a Gaussian kernel, then the results depend very much on the choice of its parameter. Finding its appropriate parameter is not trivial at all, and even locally adaptive methods [e.g., the method in Zelnik-manor and Perona (2004)] can easily fail (Nadler and Galun 2006; Luxburg 2007).

\section{Adaptive trajectory analysis of replicator dynamics}

A cluster can be seen as a dense (well-connected) region of the graph, which can be obtained via solving the following quadratic program:

$$
\operatorname{maximize} f(\mathbf{v})=\mathbf{v}^{\mathrm{T}} \mathbf{X} \mathbf{v}, \quad \text { s.t. } \mathbf{v} \geq \mathbf{0}, \quad \sum_{i=1}^{N} v_{i}=1 .
$$

The $N$-dimensional characteristic vector $\mathbf{v}$ determines the participation of the objects to the dense region (cluster). A larger $v_{i}$ indicates the stronger participation of object $i$ in the cluster. One efficient way to solve the quadratic program is to use replicator dynamics, a class of discrete time dynamical systems in the context of evolutionary game theory (Schuster and Sigmund 1983; Weibull 1997) which is defined as

$$
v_{i}(t+1)=v_{i}(t) \frac{(\mathbf{X v}(t))_{i}}{\mathbf{v}^{\mathrm{T}} \mathbf{X v}(t)}, \quad i=1, \ldots, N .
$$

It has been proven that the stable solutions of the replicator Eq. 3 are in one-to-one correspondence to the solutions of the objective function 2, which is always non-decreasing as we update the replicator equation (Weibull 1997). However, X should satisfy two conditions: (1) its diagonal elements are zero, and (2) its off-diagonal elements are non-negative and symmetric. The non-negativity condition is only required for the quadratic program in Eq. 2 
(i.e. not for the replicator dynamics in Eq. 3). Moreover, even if the elements of $\mathbf{X}$ are negative, one can subtract the smallest negative value from all the elements to make $\mathbf{X}$ non-negative. As it will be shown by Lemma 2, such a constant shift does not change the solution(s) of the quadratic program 2. On the other hand, on asymmetric matrices, replicator dynamics is related to the Nash equilibria of evolutionary strategic games, which do not necessarily correspond to the dominant mode(s) of the graph (Weibull 1997).

For a large enough $t, \mathbf{v}$ converges to the cluster that corresponds to the densest part (the dominant mode) of the graph. Thereby, the clustering methods based on replicator dynamics (e.g. DSC; Pavan and Pelillo 2007; Ng et al. 2012; Liu et al. 2013) perform a sequential peeling-off strategy where at each step,

1. first perform a replicator dynamics on the available graph, and

2. then construct a new cluster by separating the nodes whose characteristic values $\left(v_{i}\right)$ are higher than a cut_off parameter.

Thereby, this algorithm requires two parameters to be fixed in advance: cut_off and the number of update iterations of replicator dynamics shown by $T$. However, in general, choosing appropriate values of cut_off and $T$ is not straightforward. DSC suggests to fix cut_off with the epsilon of the programming language used for implementation, e.g. 2.2204e - 16. It also proposes to run the replicator dynamics long enough until it converges, i.e. $T$ could be a very large number.

\subsection{Trajectory of replicator dynamics}

Dominant Set Clustering performs the replicator dynamics until it converges to a stable solution. However, this strategy yields several deficiencies:

1. Setting appropriate values for $T$ and cut_off can be very difficult, and it might require prior knowledge about the underlying structure.

2. The convergence of replicator dynamics might be slow, i.e. it might require even an exponential number of iterations (w.r.t. the input size) to converge. Particularly, for asymmetric structures, the replicator dynamics quickly separates the far away clusters, but, then, only very slowly can discriminate the closer structures.

3. DSC might split a perfect cluster from the middle, due to e.g. choosing an inappropriate $T$ or cut_off.

Figure 1 demonstrates these issues for a simple dataset, where the pairwise similarities are computed by $\mathbf{X}_{i j}=\max (\mathbf{D})-\mathbf{D}_{i j}+\min (\mathbf{D})$ and $\mathbf{D}$ is the matrix of pairwise squared Euclidean distances. We observe that DSC requires a rather large number of iterations to separate perfectly the clusters (the smallest $T$ is 130 as shown in Fig. 1d). If we stop earlier, either the close clusters are grouped together (for $T=50$ in Fig. 1b) or an inappropriate cut is performed (for $T=125$ in Fig. 1c), which is due to the fixed value of the cut_off parameter. Thus, finding the optimal threshold for both $T$ and cut_off parameters, to separate exactly the most dominant cluster, can be very challenging or even impossible. On the other hand, this procedure is rather slow and computationally very expensive.

An important source of information unused by DSC is the 'trajectory' of $\mathbf{v}$ at different steps of replicator dynamics, which reveals informative transitions. Figure 2 shows the content of $\mathbf{v}$ at different update steps (i.e., $T=10,50,125,130$ ). After only few updates, e.g. $T=10$, already a transition occurs among the $v_{i}$ 's of different objects. A transition refers to an abrupt change in $\mathbf{v}$, if the $v_{i}$ 's are ordered, i.e. there exist two consecutive elements in ordered $\mathbf{v}$ which differ significantly compared to the other consecutive elements. At the point of transition, 
(a)

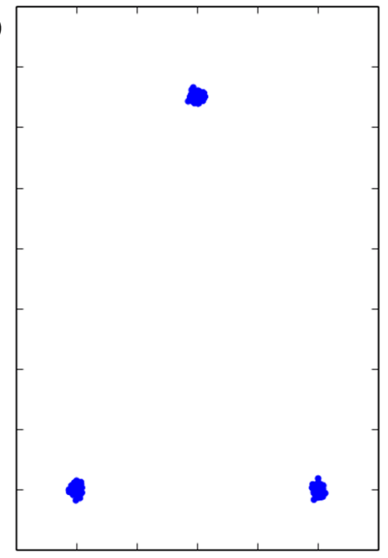

(c)

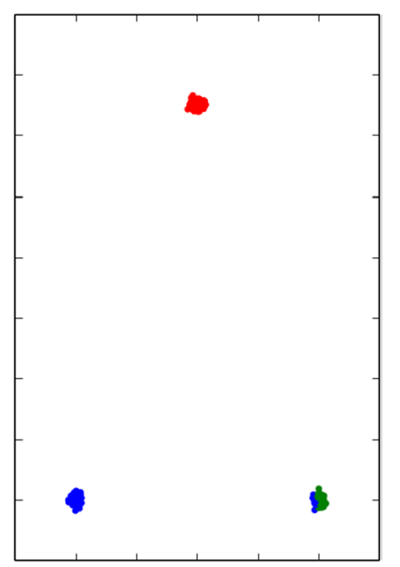

(b)

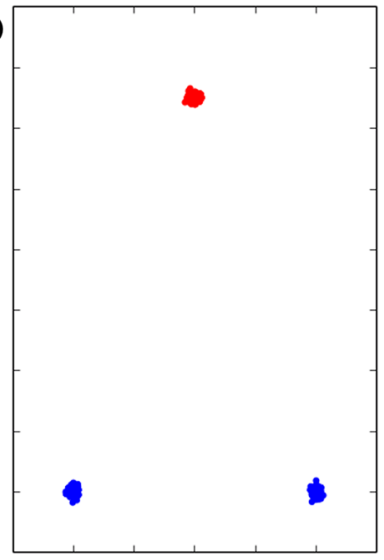

(d)

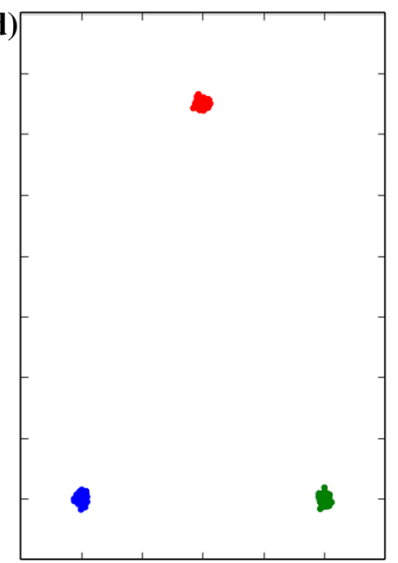

Fig. 1 DSC clustering solutions for different number of updates of replicator equation. DSC might require a large number of iterations even for a rather simple dataset to compute appropriate clusters. $\mathbf{a} T=10, \mathbf{b} T=50$, c $T=125$ and $\mathbf{d} T=130$

we observe the separation of a subset of data (the cluster at the top) from the rest of dataset. More precisely, we observe that replicator dynamics very quickly converges locally within the clusters, while different clusters have different $v_{i}$ 's. ${ }^{1}$ This behavior is comparable with power iteration, a rather similar iterative procedure used to compute the largest eigenvector and is used and analyzed by PIC (Lin and Cohen 2010). Then, based on this analysis, we adopt the local, i.e. cluster-level, convergence of replicator dynamics before its global convergence to the mode of the graph. Thus, a sharp transition does not split a valid cluster from the middle. Note that PIC runs power iteration only once, which, as we will discuss later, is not sufficient to capture all of the clusters.

Thereby, a limited number of steps (a small $T$ ) might not be enough to specify convergence to a single cluster precisely. Therefore, this information cannot be used by the standard DSC algorithm as it waits until the replicator dynamics converges to the most dominant cluster

1 The functionality of replicator dynamics and appearance of sharp transitions is independent of the size of the clusters, which is particularly due to the normalization term in the denominator. We have repeated this experiment with 6000 objects instead of 300 objects, the results are very much consistent. 

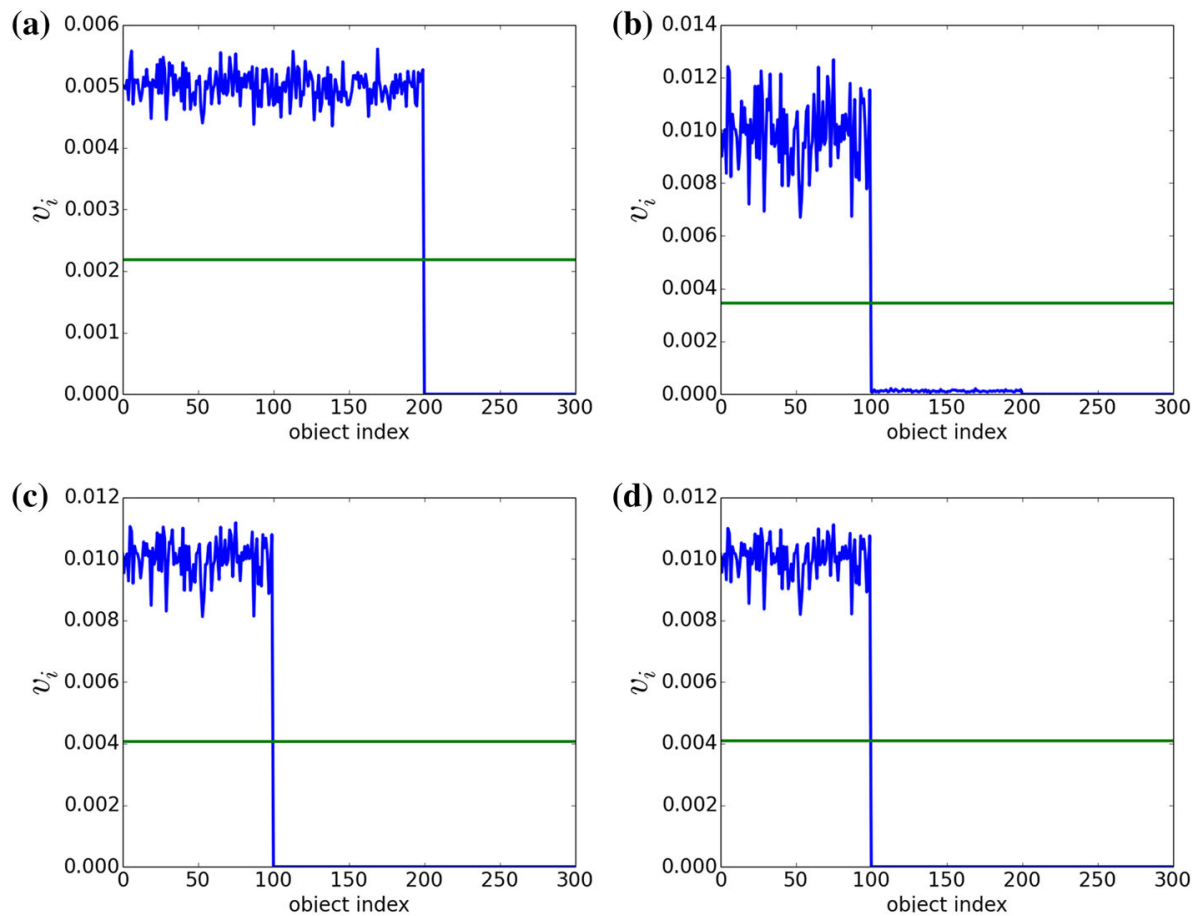

Fig. 2 Trajectory analysis of replicator dynamics: it reveals informative transitions while updating. A sharp transition corresponds to a cut over the dataset. a $T=10, \mathbf{b} T=50, \mathbf{c} T=125$ and $\mathbf{d} T=130$

(earliest occurrence happens at $T=130$ ). In summary, this analysis provides interesting insights on the performance of replicator dynamics:

1. During running a replicator dynamics, it very quickly converges locally within the clusters and then converges globally to the mode of graph. This observation can be verified in the same way as for power iteration (Lin and Cohen 2010). Thus, several transitions might occur which provide important information.

2. Since after few iterations, $v_{i}$ 's become cluster-wise almost constant, thus a transition represents a cut over the dataset, as we observe in Fig. 2. This is consistent with the final value of $\mathbf{v}$ wherein $v_{i}$ is nonzero for the objects belonging to the mode (densest region) of the graph and otherwise it is zero.

3. Appearance of a transition (which identifies a valid cut over the data) is much cheaper (faster) than separating the mode cluster and thereby waiting until the convergence of the replicator dynamics.

4. The induced cuts are independent of the size of clusters, e.g. if we double the size of clusters, the transition will still occur between different clusters due to cluster-level convergence.

We employ these insights to design an efficient algorithm called Replicator Dynamics Clustering $(R D C)$.

\subsection{Efficient detection of transitions}

Our clustering algorithm will rely on detecting the (sharpest) transition on (ordered) $\mathbf{v}$ which identifies a cut over dataset. The sharpest transition means, in a sorted variant of $\mathbf{v}$ (called $\mathbf{u}$ ) 
we obtain the pair of consecutive objects which have the maximal difference, i.e.

$$
\text { sharp_trans }=\max _{i}\left|u_{i+1}-u_{i}\right|, \quad 1 \leq i \leq N-1 .
$$

Thereby, to compute the sharpest transition, a naive approach is to prepare an increasingly sorted copy of $\mathbf{v}$ (i.e. $\mathbf{u}$ ), compute the difference between each consecutive pair of sorted elements and then choose the maximal difference (gap), which represents the maximal transition. Then, the middle of this gap gives the most distinguishing cut_off. Sorting the $N$ elements of $\mathbf{v}$ requires $O(N \log N)$ running time. However, for computing the most dominant transition, we do not need to sort the whole vector, since identifying the exact positions of many items is not relevant. Thereby, we propose a more efficient algorithm which is linear in $N$ (Algorithm 1). We first divide the interval $[\min (\mathbf{v}), \max (\mathbf{v})]$ into $N-1$ equal-size blocks, where the size of each block is $h=(\max (\mathbf{v})-\min (\mathbf{v})) /(N-1)$. We assign the objects to the blocks, such that the block of object $i$ is determined by $b[i]=\left\lfloor\frac{\left(v_{i}-\min (\mathbf{v})\right)}{h}\right\rfloor$. Then, we construct list $\mathbf{L}$ by concatenating the minima and the maxima of the blocks in order, i.e.

$$
\begin{aligned}
\mathbf{L}= & \left\{\min (\mathbf{v}), \min \left(b_{1}\right), \max \left(b_{1}\right), \ldots, \min \left(b_{k}\right), \max \left(b_{k}\right),\right. \\
& \left.\ldots, \min \left(b_{N-1}\right), \max \left(b_{N-1}\right), \max (\mathbf{v})\right\} .
\end{aligned}
$$

Note that $\mathbf{L}$ is increasingly sorted. Finally, we compute the difference between each consecutive pair in $\mathbf{L}$ to obtain the maximal transition (gap), whose middle gives the cut_off. Lemma 1 guarantees the correctness of this algorithm.

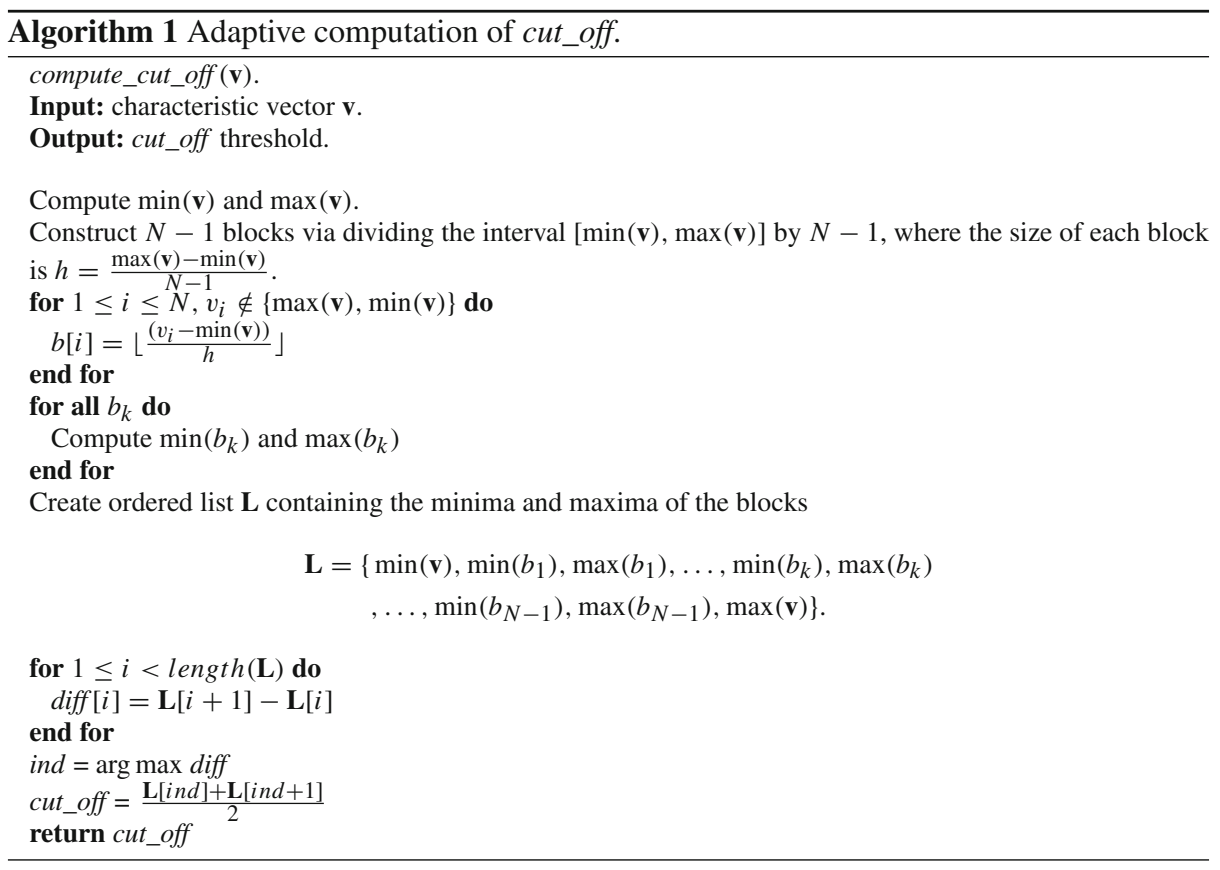

Lemma 1 Algorithm 1 computes the sharpest transition on a given vector $\mathbf{v}$.

Proof The correctness of this algorithm is proven via the Pigeonhole principle: after picking out $\min (\mathbf{v})$ and $\max (\mathbf{v})$, we construct $N-1$ blocks, but there are at most $N-2$ elements 
left from v. Therefore, according to the Pigeonhole principle, at least one block is left empty. This implies that the largest gap is at least $h$, the size of blocks. Therefore, the largest gap cannot happen inside a block. Thus, we only need to consider the difference of $\max \left(b_{k-1}\right)$ and $\min \left(b_{k}\right)$ and ignore the other elements of the blocks.

Computational complexity Assigning the elements to blocks as well as computing the blockwise min and max operations are linear. Therefore, the total computational complexity is $O(N)$ instead of $O(N \log N)$.

\subsection{Replicator dynamics clustering (RDC)}

The analysis of the trajectory of replicator dynamics at different steps inspires an efficient iterative clustering algorithm: A replicator dynamics, very quickly, reveals a transition, which corresponds to a cut over the data. As mentioned earlier, the reason is that replicator dynamics very quickly tends to be constant cluster-wise. With this observation, the choice of parameter $T$ then is not critical since choosing a large enough $T$ any way renders a valid transition, i.e. $v_{i}$ 's are still cluster-wise constant. The ultimate transition, i.e. if $T$ is selected to be very large, separates the mode of the graph from the rest.

We exploit such observations to design an efficient algorithm based on identifying the transitions in (ordered) v. Our algorithm uses three main data structures:

1. List_of_Cuts: keeps the list of (potential) clusters. Essentially, it is a list of lists, where each list List_of_Cuts[k] represents a subset of data containing a cluster or a collection of clusters.

2. Splits: is a list of lists where each list Splits $[k]$ contains the bi-partitioning of the subset stored in List_of_Cuts[k] via performing a replicator dynamics.

3. gain: stores the improvement in the objective function $f(\mathbf{v})$ if we split List_of_Cuts[k] into two subsets (clusters) Splits[k][1] and Splits[k][2].

The clustering procedure is established in two steps: (1) Cut Identification, and (2) Cluster Pruning. As we will see later, such a procedure provides automatic separation of structure from noise and outliers.

\subsubsection{Cut Identification}

At the first step, we partition the data space by performing the important cuts via replicator dynamics. We compute the cuts in a top-down manner. At the beginning the data includes no cut. Then, iteratively, the most significant cut is selected and performed at each step. The procedure continues for no_of_clusters -1 cuts, such that at the end, there will be no_of_clusters subsets of original data. The significance of a cut is determined via the amount of the gain obtained from performing the cut, i.e. how much the objective function $f(\mathbf{v})$ increases if we perform the cut. For this purpose, we investigate the impact of performing a replicator dynamics on each list in List_of_Cuts. We calculate the gain $g$ of splitting the $k$ th list (cluster) as the difference between the objective function of the completed replicator dynamics (after $T$ updates) and the objective function of a uniform $\mathbf{v}$ (i.e. when the $v_{i}$ 's are the same) over the members of that list, i.e.

$$
g_{k}=f(\mathbf{v}(T))-f\left(\mathbf{v}\left(t_{0}\right)\right) .
$$


$f\left(\mathbf{v}\left(t_{0}\right)\right)$ represents the value of the objective function when we have a uniform distribution over $\mathbf{v}$ (the initial $\mathbf{v}$ ) and is obtained by

$$
f\left(\mathbf{v}\left(t_{0}\right)\right)=\frac{1}{\operatorname{dim}(\mathbf{X})} \mathbf{e}^{\mathrm{T}} \mathbf{X} \frac{1}{\operatorname{dim}(\mathbf{X})} \mathbf{e}=\frac{\operatorname{sum}(\mathbf{X})}{\operatorname{size}(\mathbf{X})} .
$$

e is a vector of $1 \mathrm{~s}$, and $\operatorname{sum}(\mathbf{X})$ and $\operatorname{size}(\mathbf{X})$ respectively indicate the sum and the number of elements of $\mathbf{X}$. We rank the splits of current subsets of data according to their $g_{k}$ 's and choose the one which yields the maximal gain. Then, we pop out the respective list from List_of_Cuts (i.e. List_of_Cuts[maxInd]) and instead, replace its sub-lists (the new potential clusters) stored in Splits[maxInd][1] and Splits[maxInd][2]. We update the other data structures too, i.e. we perform replicator dynamics on Splits[maxInd][1] and Splits[maxInd][2] and then replace Splits[maxInd] by the resultant splits. The vector gain is also updated accordingly. Algorithm 2 describes the procedure in detail.

In Algorithm 2, we apply the cutting for a fixed number of clusters no_of_clusters. In this way we are consistent with most of the other clustering methods, which require fixing the number of clusters in advance. However, in our approach, we always sort and prioritize the potential cuts according to their respective gain and at each step pick the cut with maximal gain. Thus, instead of fixing the number of clusters in advance, one can continue the cutting procedure until a desired resolution is attained, specified by gain. Even, one can perform the cutting for different gain-thresholds to investigate how well the clusters appear at different levels (resolutions), and then provide a multi resolution data exploratory analysis.

\subsubsection{Cluster Pruning}

So far, our method identifies the main cuts over the dataset. However, the cuts might be contaminated by outliers and noise. An example is depicted in Fig. 3, where Fig. 3a shows the cuts performed by Algorithm 2. Therefore, at the next step, we prune the clusters to separate the structure from noise. In Algorithm 2, the lists stored in Splits contain the bipartitioning of the subsets (clusters) in List_of_Cuts. Whenever there is only one cluster in List_of_Cuts[k], the bi-partitioning then separates the well-connected part (i.e. the structure) from the rest (i.e. the noise). Thus, for Cluster Pruning we keep Split [k][1] and report Split $[k][2]$ as noise. Figure $3 b$ shows the results of applying this pruning step. Note that methods like $K$-means or spectral methods might produce results similar to Fig. 3a, i.e. they do not separate structure from the noise.

(a)

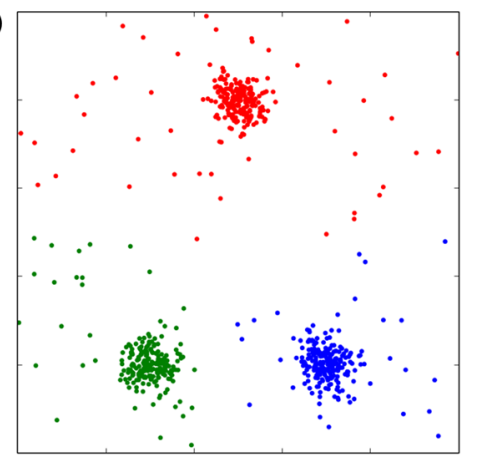

(b)

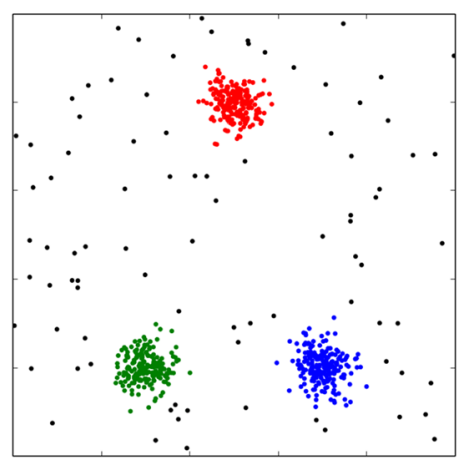

Fig. 3 Steps of Algorithm 2. a Cut Identification step and b Cluster Pruning step 


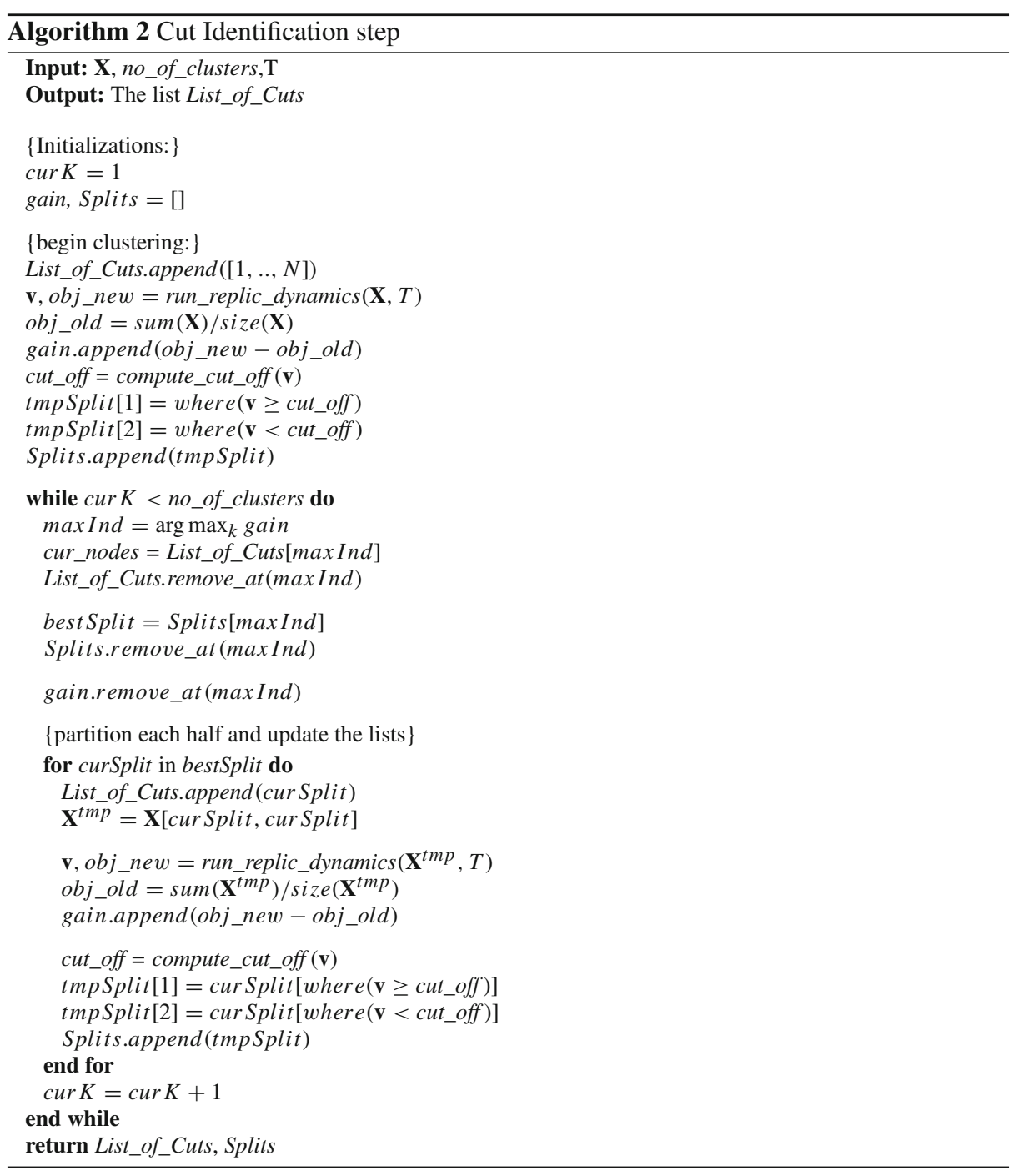

Thereby, our clustering approach provides two main advantages:

1. There will be no need to fix cut_off in advance, since it is determined automatically.

2. The choice of $T$ is not critical any more (a large enough $T$ is sufficient), since anyway a large enough $T$ will represent a valid transition and cut.

Notice that the order of the cuts represented by different large enough $T$ 's might differ as shown in Fig. 2a, b, but both are valid cuts. In this figure, the choice of $T=10$ or $T=50$ does not lead to different final clustering solutions, only the order of the cuts changes.

\subsection{Adaptive replicator dynamics}

Although the formation of a transition occurs faster than the convergence of the replicator dynamics to its stable solution, however, we can still accelerate the occurrence of transition. In 
(a)

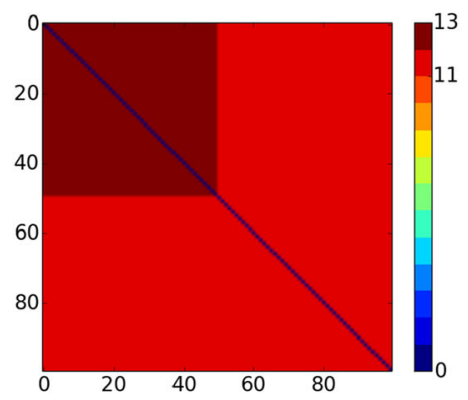

(c)

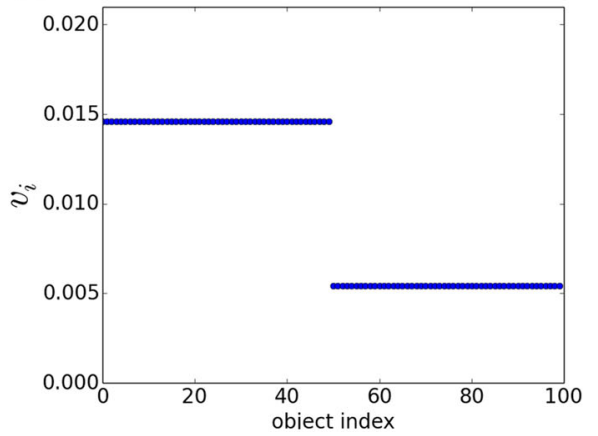

(b)

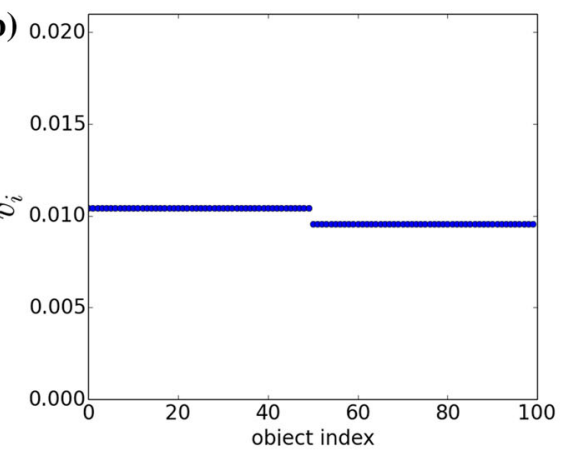

(d)

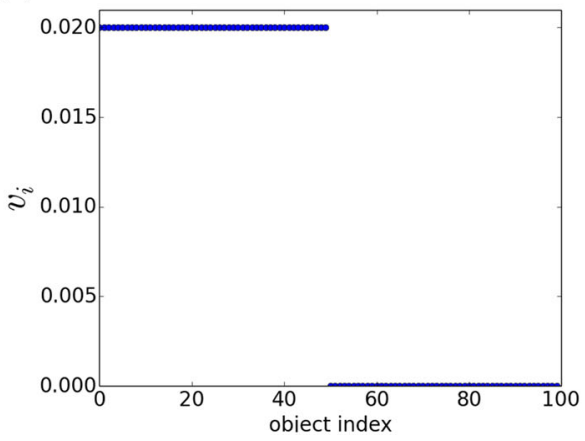

Fig. 4 Replicator dynamics might converge slowly when the structure is nested. The regularized replicator dynamics yields a faster occurrence of transition and convergence to a stable solution. a Dataset, $\mathbf{b} T=1$, c $T=10$ and $\mathbf{d} T=40$

particular, we observe that when the data contains nested structures, then, (1) the convergence to the deepest structure might be slow, and (2) the sharp transition separating the nested structure from the rest of data might occur only after a large $T$. A toy example is depicted in Fig. 4, where the pairwise similarities of the interior structure are fixed at 13 and the other similarities are 11 (Fig. 4a). The convergence of the replicator dynamics is rather slow i.e. it takes $T=40$ update steps to reach an optimal stable $\mathbf{v}$, as shown in Fig. $4 \mathrm{~d}$. At the intermediate steps, i.e. $T=1$ (Fig. 4b) or $T=10$ (Fig. 4c) the transition might not be strong (sharp) enough yet to indicate a cut.

Essentially, a sharp transition occurs whenever a subset of objects contribute more than the others to the characteristic vector, i.e. their $v_{i}$ 's are significantly larger. Therefore, in order to accelerate the appearance of sharp transitions, we propose to add the regularization term $\lambda|| \mathbf{v} \|_{2}^{2}$ to the objective function, to force a tighter distribution over $\mathbf{v}$. Thus, we replace $f$ in Eq. 2 by $f^{r e g}$ defined as

$$
f^{r e g}(\mathbf{v}, \lambda)=\mathbf{v}^{\mathrm{T}} \mathbf{X} \mathbf{v}+\lambda\|\mathbf{v}\|_{2}^{2}
$$

A similar regularization but with an opposite sign has been introduced in Pavan and Pelillo (2003), which is used for hierarchical clustering. In that context, some bounds on the regularizer are obtained via an eigen analysis that might be computationally expensive. In our setup, as we will see, we compute the optimal $\lambda$ in a closed-form way through solving 
the new objective function via replicator dynamics. First, similar to Pavan and Pelillo (2003), we introduce Lemma 2.

Lemma 2 The solution( $s$ ) of the quadratic program 2 are invariant w.r.t. shifting all elements of matrix $\mathbf{X}$ by a constant.

Proof By shifting the elements of $\mathbf{X}$ by $\lambda$, the objective function in 2 is written by

$$
f(\mathbf{v}, \lambda)=\mathbf{v}^{\mathrm{T}}\left(\mathbf{X}+\lambda \mathbf{e e}^{\mathrm{T}}\right) \mathbf{v} .
$$

Then, we have,

$$
\begin{aligned}
\mathbf{v}^{\mathrm{T}}\left(\mathbf{X}+\lambda \mathbf{e e}^{\mathrm{T}}\right) \mathbf{v} & =\mathbf{v}^{\mathrm{T}} \mathbf{X} \mathbf{v}+\mathbf{v}^{\mathrm{T}} \lambda \mathbf{e e}^{\mathrm{T}} \mathbf{v} \\
& =\mathbf{v}^{\mathrm{T}} \mathbf{X} \mathbf{v}+\lambda \underbrace{\left(\mathbf{v}^{\mathrm{T}} \mathbf{e}\right)}_{=1} \underbrace{\left(\mathbf{e}^{\mathrm{T}} \mathbf{v}\right)}_{=1} \\
& =\mathbf{v}^{\mathrm{T}} \mathbf{X} \mathbf{v}+\lambda, \quad
\end{aligned}
$$

where $\mathbf{e}=(1,1, \ldots, 1)^{\mathrm{T}}$ is a vector of $1 \mathrm{~s}$. Therefore, shifting the elements of $\mathbf{X}$ dose not change the optimal solution(s) of 2 .

Theorem 1 There is an one-to-one correspondence between the solutions of the quadratic program 8 and the replicator dynamics acting on $\mathbf{Y}$ defined as

$$
\mathbf{Y}=\mathbf{X}-\lambda\left(\mathbf{e e}^{T}-\mathbf{I}\right)
$$

Proof We first expand the regularized objective function 8 in a similar way to the regularized objective function in Pavan and Pelillo (2003).

$$
\begin{aligned}
\mathbf{v}^{\mathrm{T}} \mathbf{X} \mathbf{v}+\lambda\|\mathbf{v}\|_{2}^{2} & =\mathbf{v}^{\mathrm{T}} \mathbf{X} \mathbf{v}+\lambda \mathbf{v}^{\mathrm{T}} \mathbf{v} \\
& =\mathbf{v}^{\mathrm{T}}(\mathbf{X}+\lambda \mathbf{I}) \mathbf{v} .
\end{aligned}
$$

However, the replicator dynamics 3 cannot be directly applied to matrix $\mathbf{v}^{\mathrm{T}}(\mathbf{X}+\lambda \mathbf{I}) \mathbf{v}$, as its diagonal elements are non-zero (violation of condition I). To make the diagonal elements zero, we replace $\mathbf{v}^{\mathrm{T}}(\mathbf{X}+\lambda \mathbf{I}) \mathbf{v}$ by

$$
\mathbf{v}^{\mathrm{T}}\left(\mathbf{X}+\lambda \mathbf{I}-\lambda \mathbf{e} \mathbf{e}^{\mathrm{T}}\right) \mathbf{v}
$$

i.e. we shift all elements of $\mathbf{v}^{\mathrm{T}}(\mathbf{X}+\lambda \mathbf{I}) \mathbf{v}$ by $-\lambda$. This transformation is valid, as according to Lemma 2, shifting all the elements by a constant does not change the solution of the objective function. Thereby we obtain matrix $\mathbf{Y}$ defined as

$$
\mathbf{Y}=\mathbf{X}-\lambda\left(\mathbf{e e}^{\mathrm{T}}-\mathbf{I}\right),
$$

on which performing the replicator dynamics gives the solutions of the regularized objective function 8 .

Optimal regularization Choosing a large $\lambda$ might be interesting as it renders a tighter distribution on $\mathbf{v}$, which yields quicker appearance of a sharp transition. However, there is an upper limit on the value of $\lambda$. Theorem 2 determines such an upper bound on $\lambda$.

Theorem 2 The largest $\lambda$ that can be used to accelerate the appearance of a sharp transition is the minimum of the off-diagonal elements of $\mathbf{X}$. 

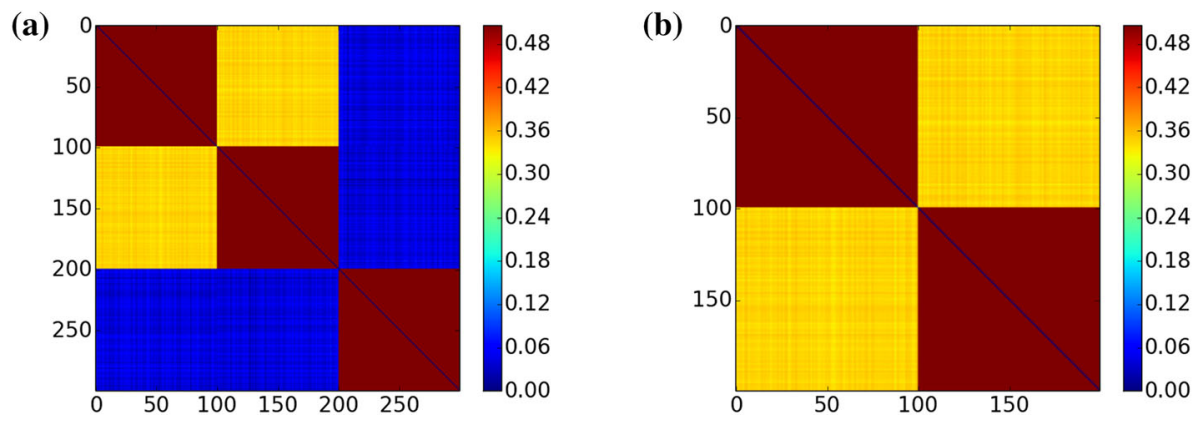

Fig. 5 The similarity matrix of the dataset in Fig. 1 and the similarity matrix of the second half. a Whole dataset and $\mathbf{b}$ second half

Proof Adding $-\lambda\left(\mathbf{e e}^{\mathrm{T}}-\mathbf{I}\right)$ to $\mathbf{X}$ implies shifting the off-diagonal elements of $\mathbf{X}$ by $-\lambda$. According to condition II, the off-diagonal elements must be non-negative. Thus, there is a limit on the negative shift of off-diagonal elements. i.e. the largest negative shift is the minimum of off-diagonal elements.

Therefore, inside the run_replic_dynamics $(\mathbf{X}, T)$ function, we first subtract the offdiagonal elements of $\mathbf{X}$ by their minimum and then perform the replicator dynamics for $T$ update steps. Using this adaptive replicator dynamics on the toy dataset of Fig. $4 \mathrm{a}$, we obtain the sharpest transition and its convergence only after one update step, i.e. for $T=1$, which is significantly smaller than $T=40$ for the unregularized version (Fig. 4). In our running example (the dataset depicted in Fig. 1), after performing the first cut, i.e. separating the cluster at the top, the second half of the dataset contains the two lower clusters. The matrix of pairwise similarities for the whole data as well as for the second half are depicted in Fig. 5. The similarity matrix of the second half shows two nested clusters, although the inter-cluster similarities are non-zero. The non-zero inter-cluster similarities render the convergence of replicator dynamics slow. The regularized objective function 8 makes the inter-cluster similarities zero, thereby accelerates the convergence and also the occurrence of a sharp transition. Using adaptive replicator dynamics, we need a smaller $T$, e.g. $T=15$ instead of $T=40$ when using Replicator Dynamics Clustering. Remember that for the standard Dominant Set Clustering we need at least $T=130$.

In general, the objects that have small similarities to the other objects are those which belong to the cluster(s) that are far from the other clusters (e.g. the top cluster in the dataset of Fig. 1). Then, these objects (clusters) correspond to the sharpest transition. Thus, in our approach, the cut that splits the data, also breaks the smallest pairwise similarities. Hence, the remaining pairwise similarities in each side are considerably larger, compared to the broken similarities. Thereby, shifting the off-diagonal similarities in each half can speed up the occurrence of new transitions. The more far away some clusters or objects are from the others, the faster/sharper the transition happens. Then, the smallest similarities are broken (cut) and thus shifting can be even more helpful since the difference between the broken similarities and the similarities in each side is even more significant. This situation happens in particular when the clusters are positioned in an asymmetric way, which is a property of large real datasets. However, in very high-dimensional data, the objects tend to lie on the surface of a hypersphere, which yields very large and at the same time similar pairwise distances. Thus, the proposed regularization strategy might be less effective in this setting. Nevertheless, as it has been analyzed for example in Barkai and Sompolinsky (1994), Buhmann et al. (2012), 
the clustering task is not well defined and relevant in this setting, since the clusters are not sufficiently distinguishable.

\section{Experiments}

We investigate the effectiveness of our algorithm on a variety of synthetic and real-world datasets and compare the results against recent clustering methods.

\subsection{Experiments with synthetic data}

First, we study the different aspects of RDC compared to the alternative methods.

Sensitivity to outliers It is known that spectral methods (e.g. SC, PIC and PSC) are sensitive to the presence of outliers (Rahimi and Recht 2004; Nadler and Galun 2006). For example, spectral methods are not able to separate the signal from the noise for the data depicted in Fig. 3. RDC does this separation because of its inherent property which aims at capturing the well-connected regions of the data.

Clusters with arbitrary shapes An advantage of spectral methods for clustering is supposed to be the ability to cope with the arbitrary shape of clusters. However, this ability depends very much on the particular choice of pairwise similarities, particularly on the choice of $\sigma$ when $\mathbf{X}_{i j}=\exp \left(-\mathbf{D}_{i j} / \sigma\right)$ (Nadler and Galun 2006; Luxburg 2007) such that finding an appropriate value for $\sigma$ is not trivial at all and requires prior knowledge about the shape and the type of clusters, which is not practical in many applications. An effective approach to extract clusters with arbitrary shapes is to use Path-based distance measure (Fischer and Buhmann 2003), which computes the minimum largest gap among all admissible paths between the pairs of objects. This choice is non-parametric, i.e. it does not need any specific parameter choice. Combining our method with this distance measure makes a lot of sense: Path-based measure essentially computes the transitive (indirect) relations, i.e. maps a cluster with an arbitrary shape to a well-connected sub-graph. Our algorithms provides an efficient way to extract significant well-connected groups.

Figure 6 illustrates the results on two circular datasets. We obtain the Path-based distances $\mathbf{D}^{\text {path }}$ from the pairwise squared Euclidean distances $\mathbf{D}$. We, then, compute $\mathbf{X}$ by $\mathbf{X}=$ $\max \left(\mathbf{D}^{\text {path }}\right)-\mathbf{D}^{\text {path }}+\min \left(\mathbf{D}^{\text {path }}\right)$ and apply RDC. Thus, no parameter is fixed in advance to compute the pairwise similarities correctly.

A general view: RDC versus PIC Both RDC and PIC perform based on an interesting observation: an equation, e.g. power iteration (PIC) or replicator dynamics (RDC), updates an initial vector iteratively, which quickly converges locally within the clusters and then converges globally either to a fixed vector representing the largest eigenvector (PIC) or to the mode of graph (RDC). Therefore, early stopping the procedure helps identification of clusters. However, a fundamental difference is that RDC performs multiple replicator dynamics sequentially; while PIC runs power iteration only once, thereby yields one clustering indicator vector. We demonstrate that providing only one indicator vector is not enough to capture all structures.

We consider a dataset containing three well-separated and spherical Gaussian clusters (shown in Fig. 7a), where the first cluster (indices: 1..100) is far from the two others (indices: 101..300). For such cases, PIC is not able to discriminate the low level structures as shown in 
(a)

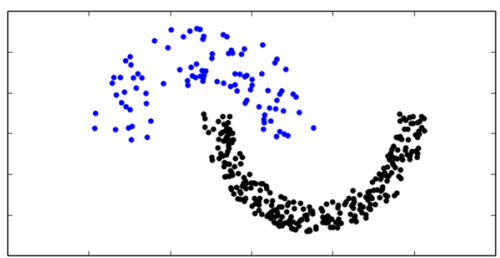

(b)

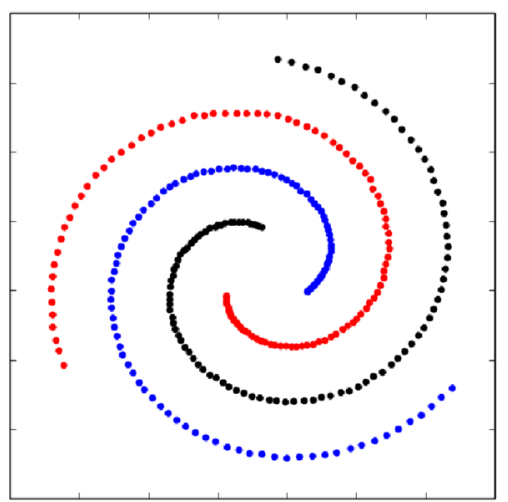

Fig. 6 Combination of path-based measure with RDC to cluster data with arbitrary clusters. a Two-moons dataset and $\mathbf{b}$ spiral dataset

(a)

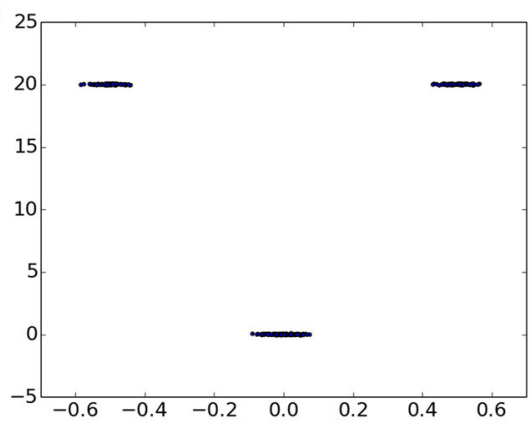

(b)

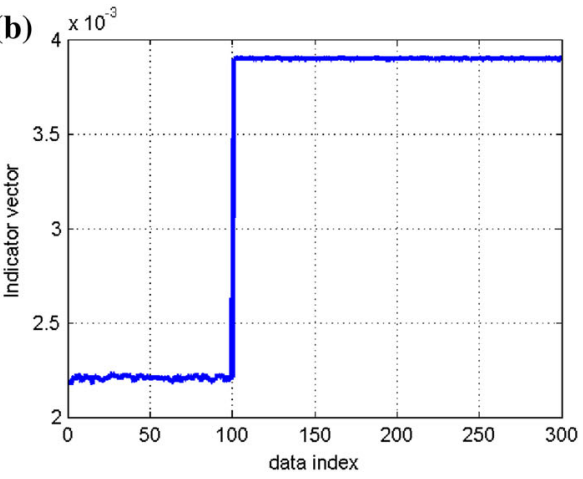

Fig. 7 Analysis of PIC when one cluster stays far away from the two other clusters. Power iteration is not able to distinguish the finer resolution. a Dataset and $\mathbf{b}$ power iteration vector

Fig. 7b. The cluster indicator vector is almost the same for the second and third clusters. This observation is consistent with the behavior of power iteration which essentially approximates only an one-dimensional embedding for the data. RDC overcomes this issue by detecting the transitions of the characteristic vector and repeating replicator dynamics for each subset. Such an analysis can also be applied to PIC to improve the results. However, replicator dynamics has advantages over power iteration: (1) it efficiently extracts the significant subsets and separates signal from the noise, (2) there is a direct interpretation of the replicator dynamics in terms of an objective function whose regularization to represent different resolutions can be integrated into the similarity matrix, thereby replicator dynamics becomes still usable, and (3) the number of update steps, i.e. $T$, is not very critical for replicator dynamics as it is for power iteration. By choosing a large $T$, power iteration might loose a transition as it ultimately converges to a constant vector. However, replicator dynamics converges to the mode of graph, thereby an unnecessary large $T$ will still indicate a sharp transition.

\subsection{Real-world experiments}

We investigate the performance of clustering methods on several real-world datasets from different domains. 
Data We compare the methods on six real-world datasets. The first three datasets are selected from the 20 newsgroups text collection (Mitchell 1997):

1. 20ngA: includes the documents of 'misc.forsale', 'soc.religion.christian' and 'talk.politics.guns'.

2. 20ngB: includes the documents of 'misc.forsale', 'soc.religion.christian', 'talk.politics.guns' and 'rec.sport.baseball'.

3. 20ngC: includes the documents of 'rec.motorcycles', 'sci.space', 'talk.politics.misc' and 'misc.forsale'.

Two datasets come from MNIST digit datasets (LeCun et al. 1998):

4. MnstA: contains the digit images of 3,5 and 7.

5. MnstB: contains the digit images of 2, 4, 6 and 8 .

The last dataset is selected from Yeast dataset of UCI repository:

6. Yeast: contains the following classes: 'CYT', 'NUC', 'MIT' and 'ME3'.

For each dataset, we compute the pairwise cosine distances between the pairs of objects and then apply the Path-based distance measure. Finally, we convert the pairwise distances to similarities (by negation and shift) to obtain the similarity matrix $\mathbf{X}$.

Alternative methods We compare RDC against five recent clustering methods: (1) Spectral Clustering (SC), (2) Power Iteration Clustering (PIC), (3) P-Spectral Clustering (PSC), (4) Dominant Set Clustering (DSC), and (5) InImDyn.

The enumeration method in Bulò et al. (2009) although is expected to work in theory, but it fails in practice: after adapting the similarity matrix w.r.t. the first equilibrium, the new replicator dynamics still converges to the first equilibrium. The method in Liu et al. (2013) has some limitations: (1) The method is particularly good when the graph is sparse and contains many small clusters, where the expansion step does not occur often, i.e. updating the solution to represent the whole graph is not necessary. However, in many clustering applications, we need to extract few but large clusters, where this method becomes less efficient, even compared to the Dominant Set Clustering algorithm. (2) The method requires setting some additional parameters where the results might be very sensitive to them. (3) Finally, our method, i.e. RDC, can be integrated into this method, particularly to the shrink step where a replicator dynamics is performed on a subset of the graph until its convergence, which might be itself inefficient and slow.

Evaluation criteria The true labels of the objects, i.e. the ground truth, are available. Thereby, we can evaluate the quality of the clusters by comparing against the ground truth. We compute three quality measures: (1) adjusted Rand score (Hubert and Arabie 1985), which computes the similarity between the predicted and the true clusterings, (2) adjusted Mutual Information (Xuan Vinh et al. 2010), which measures the mutual information between two partitionings, and (3) V-measure (Rosenberg and Hirschberg 2007), which computes the harmonic mean of homogeneity and completeness. We compute the adjusted version of these criteria, such that they yield zero for random partitionings.

Results In Tables 1, 2 and 3, we demonstrate and compare the results of different methods respectively w.r.t. adjusted Rand score, adjusted Mutual Information and V-measure. Any positive value indicates a (partially) correct clustering. According to the results, RDC often performs equally well or better than the other methods for different evaluation criteria. In 
Table 1 Performance w.r.t. adjusted Rand score

\begin{tabular}{lllllll}
\hline Dataset & SC & PIC & PSC & DSC & InImDyn & RDC \\
\hline 20ngA & 0.2803 & $\mathbf{0 . 4 2 6 2}$ & 0.2994 & 0.3931 & 0.3814 & 0.3905 \\
20ngB & 0.1966 & 0.2557 & 0.1277 & 0.2379 & 0.202 & $\mathbf{0 . 2 6 1 3}$ \\
20ngC & 0.248 & 0.1753 & 0.1602 & 0.2175 & 0.2304 & $\mathbf{0 . 2 7 8 8}$ \\
MnstA & 0.394 & $\mathbf{0 . 5 0 1 9}$ & 0.3763 & 0.4487 & 0.4413 & 0.4879 \\
MnstB & $\mathbf{0 . 6 5 2 5}$ & 0.3918 & 0.3431 & 0.6312 & 0.6394 & 0.6169 \\
Yeast & 0.5418 & 0.4863 & 0.4149 & 0.5775 & 0.5518 & $\mathbf{0 . 6 5 2}$ \\
\hline
\end{tabular}

Table 2 Performance w.r.t. adjusted mutual information

\begin{tabular}{lllllll}
\hline Dataset & SC & PIC & PSC & DSC & InImDyn & RDC \\
\hline 20ngA & 0.3041 & 0.349 & 0.1789 & 0.3516 & 0.3285 & $\mathbf{0 . 3 6 3 2}$ \\
20ngB & 0.2586 & 0.242 & 0.1608 & 0.2517 & 0.2409 & $\mathbf{0 . 2 6 0 4}$ \\
20ngC & 0.2688 & 0.1786 & 0.2079 & 0.2887 & 0.3004 & $\mathbf{0 . 3 1 5}$ \\
MnstA & 0.3905 & $\mathbf{0 . 5 1 4 7}$ & 0.4033 & 0.4606 & 0.4576 & 0.4903 \\
MnstB & $\mathbf{0 . 5 8 7 5}$ & 0.3075 & 0.3249 & 0.5572 & 0.5306 & 0.5629 \\
Yeast & 0.5214 & 0.5357 & 0.4839 & 0.5637 & 0.5872 & $\mathbf{0 . 6 3 0 2}$
\end{tabular}

Table 3 Performance w.r.t. V-measure

\begin{tabular}{lllllll}
\hline Dataset & SC & PIC & PSC & DSC & InImDy & RDC \\
\hline 20ngA & 0.3050 & $\mathbf{0 . 4 3 4 2}$ & 0.2234 & 0.3818 & 0.342 & 0.3744 \\
20ngB & 0.2630 & 0.2719 & 0.192 & 0.2631 & 0.2503 & $\mathbf{0 . 2 8 0 7}$ \\
20ngC & 0.3005 & 0.2107 & 0.2469 & $\mathbf{0 . 3 3 6 6}$ & 0.3119 & 0.3268 \\
MnstA & 0.4087 & 0.4835 & 0.3202 & 0.4316 & 0.421 & $\mathbf{0 . 4 9 5 2}$ \\
MnstB & 0.5948 & 0.3344 & 0.3655 & 0.5704 & 0.5457 & $\mathbf{0 . 6 0 1 6}$ \\
Yeast & 0.5922 & 0.5796 & 0.4652 & 0.6271 & 0.6423 & $\mathbf{0 . 6 6 3 8}$
\end{tabular}

more than $60 \%$ of the cases, RDC gives the best results. In other cases, it is very close to best one. There is no other method which works (fairly) well on the all datasets. We particularly observe that PIC works well when there are few clusters in the dataset (20ngA and MnstA have only three clusters), but it might fail when there are many clusters. As we have analyzed, PIC is not appropriate for capturing structures represented at different resolutions, which is a property of datasets with many clusters (e.g. 20ngB and 20ngC). InImDyn is proposed to improve the runtime of DSC, however it does not improve the quality of the clusters, even sometimes decreases. This result is consistent with the investigation of the method in Bulò et al. (2011). PSC is a new method which works based on a non-linear generalization of the Laplacian matrix. However, the method provides less satisfying results compared to the alternatives, as well as it is computationally very expensive and demanding. For example, for 20ngC, PSC is almost 50 times slower than the other methods. For this dataset, the running times of different methods are (in seconds): 1.3381 (SC), 0.8716 (PIC), 61.9709 (PSC), 1.8792 (DSC), 1.0835 (InImDyn) and 1.1236 (RDC). We have performed the experiments 
under identical computational settings and conditions using an Intel machine with core i7$4600 \mathrm{U}$ and $2.7 \mathrm{GHz} \mathrm{CPU}$ and with $8.00 \mathrm{~GB}$ internal memory.

\section{Conclusion}

The analysis of trajectory of replicator dynamics at different steps reveals appearance of informative transitions that correspond to the cuts over data. We exploited this observation to design an efficient algorithms in two steps: (1) Cut Identification, and (2) Cluster Pruning. The key properties of our approach are: First, we do not require the replicator dynamics to converge, which can be very slow. Second, we obviate the need for fixing critical parameters which can affect a lot the results. In order to accelerate the occurrence of transitions, we proposed regularization of the corresponding objective function which yields to subtracting the off-diagonal elements of the similarity matrix by the minimum of off-diagonal elements. We performed extensive experiments on synthetic and real-world datasets to show the effectiveness of our algorithm compared to the alternative methods.

Acknowledgments We would like to thank Chris Dance and Andreas Krause for insightful discussions.

\section{References}

Bansal, N., Blum, A., \& Chawla, S. (2004). Correlation clustering. Machine Learning, 56(1-3), 89-113.

Barkai, N., \& Sompolinsky, H. (1994). Statistical mechanics of the maximum-likelihood density estimation. Physical Review E, 50(3), 1766.

Bühler, T., \& Hein, M. (2009). Spectral clustering based on the graph p-laplacian. In Proceedings of the 26th Annual International Conference on Machine Learning, ICML 'O9 (pp. 81-88). ACM.

Buhmann, J. M., Chehreghani, M. H., Frank, M., \& Streich, A. P. (2012). Information theoretic model selection for pattern analysis. Journal of Machine Learning Research, ICML Workshop on Unsupervised and Transfer Learning, 27, 51-65.

Bulò, S. R., Pelillo, M., \& Bomze, I. M. (2011). Graph-based quadratic optimization: A fast evolutionary approach. Computer Vision and Image Understanding, 115(7), 984-995.

Bulò, S. R., Torsello, A., \& Pelillo, M. (2009). A game-theoretic approach to partial clique enumeration. Image and Vision Computing, 27(7), 911-922.

Chan, P. K., Schlag, M. D. F., \& Zien, J. Y. (1994). Spectral k-way ratio-cut partitioning and clustering. IEEE Transactions on CAD of Integrated Circuits and Systems, 13(9), 1088-1096.

Fischer, B., \& Buhmann, J. M. (2003). Path-based clustering for grouping of smooth curves and texture segmentation. IEEE Transactions on Pattern Analysis and Machine Intelligence, 25(4), 513-518.

Hein, M., \& Bühler, T. (2010). An inverse power method for nonlinear eigenproblems with applications in 1-spectral clustering and sparse PCA. Advances in Neural Information Processing Systems, 23, 847-855.

Hofmann, T., \& Buhmann, J. M. (1997). Pairwise data clustering by deterministic annealing. IEEE Transactions on Pattern Analysis and Machine Intelligence, 19(1), 1-14.

Hou, J., \& Pelillo, M. (2013). A simple feature combination method based on dominant sets. Pattern Recognition, 46(11), 3129-3139.

Hubert, L., \& Arabie, P. (1985). Comparing partitions. Journal of Classification, 2(1), 193-218.

LeCun, Y., Bottou, L., Bengio, Y., \& Haffner, P. (1998). Gradient-based learning applied to document recognition. Proceedings of the IEEE, 86, 2278-2324.

Lin, F., \& Cohen, W. W. (2010). Power iteration clustering. In Proceedings of the 27th International Conference on Machine Learning (ICML-10), June 21-24, 2010, Haifa, Israel (pp. 655-662).

Liu, H., Jan Latecki, L., \& Yan, S. (2013). Fast detection of dense subgraphs with iterative shrinking and expansion. IEEE Transactions on Pattern Analysis and Machine Intelligence, 35(9), 2131-2142.

Luxburg, U. (2007). A tutorial on spectral clustering. Statistics and Computing, 17(4), 395-416.

MacQueen, J. (1967). Some methods for classification and analysis of multivariate observations. In L. M. Le Cam \& J. Neyman (Eds.), Proceedings of the 5th Berkeley Symposium on Mathematical Statistics and Probability (Vol. 1, pp. 281-297). Berkeley, CA: University of California Press. 
Mitchell, T. M. (1997). Machine learning (1st ed.). New York, NY: McGraw-Hill, Inc.

Nadler, B., \& Galun, M. (2006). Fundamental limitations of spectral clustering. In Advances in neural information processing systems (NIPS), pp. 1017-1024.

Ng, A. Y., Jordan, M. I., \& Weiss, Y. (2001). On spectral clustering: Analysis and an algorithm. In Advances in neural information processing systems (NIPS), pp. 849-885.

Ng, B., McKeown, M. J., \& Abugharbieh, R. (2012). Group replicator dynamics: A novel group-wise evolutionary approach for sparse brain network detection. IEEE Transactions on Medical Imaging, 31(3), 576-585.

Pavan, M., \& Pelillo, M. (2003). Dominant sets and hierarchical clustering. In 9th IEEE International Conference on Computer Vision (ICCV) (pp. 362-369).

Pavan, M., \& Pelillo, M. (2007). Dominant sets and pairwise clustering. IEEE Transactions on Pattern Analysis and Machine Intelligence, 29(1), 167-172.

Rahimi, A., \& Recht, B. (2004). Clustering with normalized cuts is clustering with a hyperplane. In ECCV workshop on statistical learning in computer vision.

Rosenberg, A., \& Hirschberg, J. (2007). V-measure: A conditional entropy-based external cluster evaluation measure. In EMNLP-CoNLL (pp. 410-420). ACL.

Roth, V., Laub, J., Kawanabe, M., \& Buhmann, J. M. (2003). Optimal cluster preserving embedding of nonmetric proximity data. IEEE Transactions on Pattern Analysis and Machine Intelligence, 25(12), 1540-1551.

Schuster, P., \& Sigmund, K. (1983). Replicator dynamics. Journal of Theoretical Biology, 100, 533-538.

Shi, J., \& Malik, J. (2000). Normalized cuts and image segmentation. IEEE Transactions on Pattern Analysis and Machine Intelligence, 22(8), 888-905.

Vinh, N. X., Epps, J., \& Bailey, J. (2010). Information theoretic measures for clusterings comparison: Variants, properties, normalization and correction for chance. Journal of Machine Learning Research, 11, 28372854.

Weibull, J. W. (1997). Evolutionary game theory. Cambridge, MA: MIT Press.

Zelnik-manor, L., \& Perona, P. (2004). Self-tuning spectral clustering. In Advances in neural information processing systems (NIPS) (Vol. 17, pp. 1601-1608). MIT Press. 